\title{
Directionality of acoustic-phonon emission in weakly confined semiconductor quantum dots
}

\author{
Juan I. Climente* and Andrea Bertoni \\ CNR-INFM S3, Via Campi 213/A, 41100 Modena, Italy \\ Guido Goldoni and Elisa Molinari \\ CNR-INFM S3 and Dipartimento di Fisica, Università degli Studi di Modena e Reggio Emilia, Via Campi 213/A, 41100 Modena, Italy
}

(Received 25 January 2007; published 28 June 2007)

\begin{abstract}
The direction of propagation of acoustic phonons emitted by electron relaxation in weakly confined, parabolic quantum dots charged with one or two electrons is studied theoretically. The emission angle strongly depends on the energy of the phonon, the dominant electron-phonon scattering mechanism (deformation potential or piezoelectric field), and the orbital symmetries of the initial and final electron states. This leads to different behaviors for phonons emitted by electrons relaxing between levels of single and coupled quantum dots. Our results establish the basis to control the direction of propagation of phonon modes triggered by transitions in quantum dot systems.
\end{abstract}

DOI: 10.1103/PhysRevB.75.245330

PACS number(s): 73.21.La, 73.61.Ey, 72.10.Di

\section{INTRODUCTION}

There is currently interest in understanding the physics of electron-acoustic phonon interaction in semiconductor quantum dots (QDs). This is motivated by the fact that acoustic phonons constitute the main source of relaxation between discrete energy levels with few-meV spacing, which is indeed the energy regime where weakly confined QD systems lie. ${ }^{1-4}$ Whereas much theoretical endeavor has been aimed at elucidating how phonon-induced electron-scattering rates depend on the QD geometry and the external fields, ${ }^{5-8}$ very few works have explicitly investigated the orientation of the emitted phonons. This is still an interesting problem: on the one hand, there is theoretical evidence that the anisotropy of electron-acoustic-phonon interaction strongly affects the scattering rate; 9,10 on the other hand, QDs may be used to force phonon propagation along a desired direction of the semiconductor lattice. ${ }^{11}$ The latter property can be of great significance for the development of phononic devices which would benefit from strong directionality, such as quantumdot-based phonon lasers.

The direction of propagation of acoustic phonons in parabolic QDs was first studied by Bockelmann, ${ }^{5}$ who considered electron-phonon coupling via the deformation potential (DP) interaction, and focused on electron scattering between states of individual QDs (intradot transitions). Nonetheless, an analysis of the physical arguments which determined the direction of propagation was missing. Moreover, recent studies have shown that piezoelectric field (PZ) interaction may actually become the dominant scattering mechanism in several experimentally relevant situations, ${ }^{8,13}$ so that it is interesting to investigate the influence of this mechanism on the direction of phonon propagation. Finally, a number of recent experiments probing carrier relaxation between the bonding and antibonding states of vertically coupled quantum dots (CQDs) have identified acoustic phonons as the main scattering source. ${ }^{3,4} \mathrm{~A}$ study on the phonon propagation direction in this kind of molecular (interdot) transitions is also lacking.

In this paper we study the emission angle of acoustic phonons due to electron scattering in single and vertically coupled QDs. The physical mechanisms leading to phonon propagation along a preferential direction are described for both intradot and interdot transitions. We show that the polar angle of emission can be tuned from almost parallel to almost perpendicular to the growth direction, by manipulating the energy of the electron transition. We also show how recently predicted results suggesting the suppression of $\mathrm{PZ}$ scattering in two-electron interdot transitions ${ }^{10}$ can be related to the direction of phonon emission in CQD systems.

\section{THEORY}

We study disk-shaped GaAs/AlGaAs QDs with confinement energy in the range of few meV. The theoretical model we use is the same as in Ref. 8. Thus we describe the singleelectron states within the effective mass and envelope function approximations. The confinement potential of the dots is modeled as a rectangular quantum well in the growth direction $z$, formed by the heterostructure band offset, and a twodimensional parabola in the $x y$ plane, which gives rise to the Fock-Darwin (FD) spectrum. ${ }^{14}$ The electron orbital state $\alpha$ can then be written as

$$
\psi_{\alpha}(x, y, z)=\phi_{n, m}(x, y) \xi_{g}(z),
$$

where $\phi_{n, m}$ is the $n$th FD orbital with azimuthal angular momentum $m$, and $\xi_{g}$ is the symmetric quantum well solution with parity $g$ with respect to the reflection about the $z=0$ plane, with $g=0$ (1) denoting even (odd) parity.

In GaAs/AlGaAs heterostructures, both semiconductor materials have similar elastic constants. Consequently, no significant phonon confinement is expected, and we can safely consider bulk phonons. Furthermore, since the interlevel spacing is in the few meV scale, optical phonons can be safely neglected, and electron-phonon coupling is in the socalled weak regime, where the Fermi golden rule suffices to describe the observed transition rates. ${ }^{1,5}$ We assume linear isotropic dispersion $\omega_{\sigma q}=c_{\sigma} q$, where $c_{\sigma}$ is the sound velocity of longitudinal acoustic ( $\sigma=\mathrm{LA})$ or transversal acoustic $(\sigma$ $=\mathrm{TA}$ ) phonon modes in the QD material, and $q$ is the modulus of the phonon momentum q. The electron-phonon interaction Hamiltonian reads 


$$
\mathcal{H}_{e-p}=\sum_{\nu \mathbf{q}} M_{\nu}(\mathbf{q})\left(b_{\mathbf{q}} e^{i \mathbf{q r}}+b_{\mathbf{q}}^{\dagger} e^{-i \mathbf{q r} \mathbf{r}}\right),
$$

where $b_{\mathbf{q}}$ and $b_{\mathbf{q}}^{\dagger}$ are the phonon annihilation and creation operators, respectively, and $M_{\nu}(\mathbf{q})$ is the scattering matrix element corresponding to the electron-scattering mechanism $\nu$ (see below). At zero temperature, the phonon emission rate by electron relaxation between an initial electron state $\left|\Psi_{i}\right\rangle$ and a final state $\left|\Psi_{f}\right\rangle$ is determined by the Fermi golden rule:

$$
\tau_{i f}^{-1}=\frac{2 \pi}{\hbar} \sum_{\nu \mathbf{q}}\left|M_{\nu}(\mathbf{q})\right|^{2}\left|\left\langle\Psi_{f}\left|e^{-i \mathbf{q r}}\right| \Psi_{i}\right\rangle\right|^{2} \delta\left(\left|E_{f}-E_{i}\right|-E_{q}\right),
$$

where $E_{f}$ and $E_{i}$ stand for the final and initial electron states energy and $E_{q}=\hbar \omega_{\sigma q}$ represents the phonon energy. It is immediate from Eq. (3) that the modulus of the phonon momentum is determined by the energy of the electron transition: $q_{0}=\left(\left|E_{f}-E_{i}\right|\right) / \hbar c_{\sigma}$. The electron-phonon scattering for a zinc-blende crystal is composed of the following contributions: ${ }^{8}$ (i) The electron-LA phonon scattering due to the deformation potential $(\nu=\mathrm{LA}-\mathrm{DP})$ :

$$
\left|M_{\mathrm{LA}-\mathrm{DP}}(\mathbf{q})\right|^{2}=\frac{\hbar D^{2}}{2 d c_{\mathrm{LA}} \Omega}|\mathbf{q}| ;
$$

(ii) the electron-LA phonon scattering due to the PZ field $(\nu=\mathrm{LA}-\mathrm{PZ})$ :

$$
\left|M_{\mathrm{LA}-\mathrm{PZ}}(\mathbf{q})\right|^{2}=\frac{32 \pi^{2} \hbar e^{2} h_{14}^{2}}{\varepsilon^{2} d c_{\mathrm{LA}} \Omega} \frac{\left(3 q_{x} q_{y} q_{z}\right)^{2}}{|\mathbf{q}|^{7}} ;
$$

(iii) the electron-TA phonon scattering due to the PZ field $(\nu=\mathrm{TA}-\mathrm{PZ})$ :

$$
\begin{aligned}
\left|M_{\mathrm{TA}-\mathrm{PZ}}(\mathbf{q})\right|^{2}= & \frac{32 \pi^{2} \hbar e^{2} h_{14}^{2}}{\varepsilon^{2} d c_{\mathrm{TA}} \Omega} \mid \frac{q_{x}^{2} q_{y}^{2}+q_{y}^{2} q_{z}^{2}+q_{z}^{2} q_{x}^{2}}{|\mathbf{q}|^{5}} \\
& -\frac{\left(3 q_{x} q_{y} q_{z}\right)^{2}}{|\mathbf{q}|^{7}} \mid .
\end{aligned}
$$

In the above expressions $D, d$, and $\Omega$ stand for the crystal acoustic deformation potential constant, density, and volume, respectively. $e$ is the electron charge, $h_{14}$ is the piezoelectric constant, and $\varepsilon$ is the static dielectric constant. Since there are two transverse phonon modes, the $\nu=\mathrm{TA}-\mathrm{PZ}$ contribution [Eq. (6)] must be accounted for twice in the sum of Eq. (3).

It follows from Eq. (3) that the scattering rates may depend on the direction of the phonon through the structure factor $\left\langle\Psi_{f}\left|e^{-i \mathbf{q r}}\right| \Psi_{i}\right\rangle$, and through the matrix elements $M_{\nu}(\mathbf{q})$. In fact, the matrix elements are dependent on the direction of the phonon for PZ interaction only, as reflected by the proportionality to the phonon momentum projections in Eqs. (5) and (6). In contrast, no such dependence is found for the DP interaction, where the matrix elements are proportional to the modulus of the momentum [Eq. (4)]. This constitutes a qualitative difference between DP and PZ mechanisms, and renders PZ scattering more sensitive to changes in the direction of propagation of the phonon.
It is convenient to rewrite Eq. (3) using spherical coordinates for the phonon momentum q $=q(\cos \theta \sin \varphi, \sin \theta \sin \varphi, \cos \varphi)$. In addition, since in our approximation the electron lateral and vertical degrees of freedom are decoupled, the structure factor can be written as a product of in-plane and vertical components, $\left|\left\langle\Psi_{f}\left|e^{-i \mathbf{q r}}\right| \Psi_{i}\right\rangle\right|^{2}=\left|G_{i f}^{(x y)}\left(q_{0}, \varphi\right)\right|^{2}\left|I_{i f}^{(z)}\left(q_{0}, \varphi\right)\right|^{2}$ (Ref. 5) where

$$
\begin{aligned}
G_{i f}^{(x y)}\left(q_{0}, \varphi, \theta\right)= & e^{i\left(m_{i}-m_{f}\right)(\theta+\pi / 2)} \int_{0}^{\infty} d \rho \rho R_{n_{i}, m_{i}}(\rho) \\
& \times R_{n_{f}, m_{f}}(\rho) J_{m_{i}-m_{f}}\left(\rho q_{0} \sin \varphi\right),
\end{aligned}
$$

$$
I_{i f}^{(z)}\left(q_{0}, \varphi\right)=\int_{0}^{\infty} d z \xi_{g_{i}} e^{-i q_{0} \cos \varphi z} \xi_{g_{f}}
$$

represent the coupling between the electron and the phonon waves in the in-plane and growth directions, respectively. These form factors depend on the electron initial and final states, as well as on the modulus of the phonon momentum $q_{0}$ and the polar angle $\varphi$. In Eq. (7), $R_{n, m}(\rho)$ is the radial part of the FD state $\phi_{n, m}(x, y),{ }^{14}$ and $J_{l}(x)$ the first-order Bessel function of order $l$. Noteworthy is that the form factors depend on the azimuthal angle of phonon emission $\theta$ only through a phase factor, that has no influence on the scattering rate calculation. This is due to the circular symmetry of parabolic QDs and implies that phonon emission is isotropic on the $x y$ plane. It is now possible to retrieve the following expressions for the phonon emission rate coming from the individual interaction mechanisms:

$$
\begin{gathered}
\tau_{i f, L A-D P}^{-1}=\frac{D^{2} q_{0}^{3}}{4 \pi \hbar c_{\mathrm{LA}}^{2} d} \int_{0}^{\pi} d \varphi \sin \varphi\left|\left\langle\Psi_{f}\left|e^{-i \mathbf{q r} \mid}\right| \Psi_{i}\right\rangle\right|^{2}, \\
\tau_{i f, L A-P Z}^{-1}=\frac{18 \pi e^{2} h_{14}^{2}}{\hbar c_{\mathrm{LA}}^{2} \epsilon^{2} d} q_{0} \int_{0}^{\pi} d \varphi \sin ^{5} \varphi \cos ^{2} \varphi\left|\left\langle\Psi_{f}\left|e^{-i \mathbf{q r} \mid}\right| \Psi_{i}\right\rangle\right|^{2}, \\
\tau_{i f, T A-P Z}^{-1}=\frac{8 \pi e^{2} h_{14}^{2}}{4 \hbar c_{\mathrm{TA}}^{2} \epsilon^{2} d} q_{0} \mid \int_{0}^{\pi} d \varphi\left(\sin ^{5} \varphi+8 \sin ^{3} \varphi \cos ^{2} \varphi\right. \\
\left.-9 \sin ^{5} \varphi \cos ^{2} \varphi\right)\left|\left\langle\Psi_{f}\left|e^{-i \mathbf{q r}}\right| \Psi_{i}\right\rangle\right|^{2} \mid,
\end{gathered}
$$

where $\theta$ has been integrated analytically. In what follows we will focus on determining the mean value of the emission angle $\langle\varphi\rangle$ with respect to the vertical axis, where $\varphi=0$ corresponds to emission along the growth direction $z$. The mean emission angle is obtained by multiplying the integrands of Eqs. (9) by $\varphi$ and dividing the result by the corresponding value of $\tau_{i f, v}^{-1}$. This gives a weighted sum over all possible emission directions: 


$$
\begin{gathered}
\left\langle\varphi_{i f, L A-D P}\right\rangle=\frac{\int_{0}^{\pi} d \varphi \varphi \sin \varphi\left|\left\langle\Psi_{f}\left|e^{-i \mathbf{q r}}\right| \Psi_{i}\right\rangle\right|^{2}}{\int_{0}^{\pi} d \varphi \sin \varphi\left|\left\langle\Psi_{f}\left|e^{-i q \mathbf{r}}\right| \Psi_{i}\right\rangle\right|^{2}}, \\
\left\langle\varphi_{i f, L A-P Z}\right\rangle=\frac{\int_{0}^{\pi} d \varphi \varphi \sin ^{5} \varphi \cos ^{2} \varphi\left|\left\langle\Psi_{f}\left|e^{-i \mathbf{q r} \mid}\right| \Psi_{i}\right\rangle\right|^{2}}{\int_{0}^{\pi} d \varphi \sin ^{5} \varphi \cos ^{2} \varphi\left|\left\langle\Psi_{f}\left|e^{-i \mathbf{q r} \mid}\right| \Psi_{i}\right\rangle\right|^{2}}, \\
\left\langle\varphi_{i f, T A-P Z}\right\rangle=\frac{\left.\left|\int_{0}^{\pi} d \varphi \varphi\left(\sin ^{5} \varphi+8 \sin ^{3} \varphi \cos ^{2} \varphi-9 \sin ^{5} \varphi \cos ^{2} \varphi\right)\right|\left\langle\Psi_{f}\left|e^{-i \mathbf{q r} \mid}\right| \Psi_{i}\right\rangle\right|^{2} \mid}{\left.\left|\int_{0}^{\pi} d \varphi\left(\sin ^{5} \varphi+8 \sin ^{3} \varphi \cos ^{2} \varphi-9 \sin ^{5} \varphi \cos ^{2} \varphi\right)\right|\left\langle\Psi_{f}\left|e^{-i \mathbf{q r} \mid}\right| \Psi_{i}\right\rangle\right|^{2} \mid} .
\end{gathered}
$$

For a single QD, we study the electron relaxation from $(n, m, g)=(0,1,0)$ to $(n, m, g)=(0,0,0)$, i.e., from the lowest $p$ to the lowest $s$ FD states (intradot transition). For CQDs, we study the relaxation from $(n, m, g)=(0,0,1)$ to $(n, m, g)$ $=(0,0,0)$, i.e., from the lowest antibonding (odd) to the lowest bonding (even) solution of the double quantum well (interdot transition). In most of the cases we consider, these are the fundamental transitions of the system (the transition from the first excited to the ground orbital state). In fact, this is often the most relevant transition since many QD applications rely on the formation of two-level systems, which are built using the two lowest-lying states, and it can be monitored, e.g., by means of pump-and-probe techniques. ${ }^{1}$ The $(0,1,0) \rightarrow(0,0,0)$ transition is a representative case of relaxation between in-plane orbitals (FD states), and similar qualitative behavior may be expected for related transitions such as $(0,2,0) \rightarrow(0,0,0)$. Likewise, the $(0,0,1)$ $\rightarrow(0,0,0)$ transition is a representative case of relaxation between vertical orbitals.

We assume $\mathrm{GaAs} / \mathrm{Al}_{0.3} \mathrm{Ga}_{0.7} \mathrm{As}$ QD structures, and use the same material parameters as in Ref. 8, namely electron effective mass $m^{*}=0.067$, band offset $V_{c}=243 \mathrm{meV}$, density $d=5310 \mathrm{~kg} / \mathrm{m}^{3}$, DP constant $D=8.6 \mathrm{eV}$, dielectric constant $\epsilon=12.9$, PZ constant $h_{14}=1.41 \times 10^{9} \mathrm{~V} / \mathrm{m}$, and sound velocity $c_{\mathrm{LA}}=4.72 \times 10^{3} \mathrm{~m} / \mathrm{s}$ and $c_{\mathrm{TA}}=3.34 \times 10^{3} \mathrm{~m} / \mathrm{s}^{15,16}$

\section{PHONON EMISSION IN SINGLE QUANTUM DOTS}

In this section we investigate the emission of phonons by electron scattering in isolated QDs. Figure 1 illustrates the phonon emission rate and mean emission angle due to the different electron-phonon interaction mechanisms, as a function of the lateral confinement of the dot. Note that the phonon energy $E_{q}$ equals the frequency of the confining parabola, $\hbar \omega_{0}$, as expected from the FD spectrum. One can see in Fig. 1(a) that, as a general trend, both DP and PZ interac- tions tend to emit phonons close to the growth direction $(\varphi$ $=0)$, but the emission angle is shifted towards the in-plane direction $(\varphi=\pi / 2)$ as the phonon energy becomes small. ${ }^{17}$

As an exception to this rule, we observe a series of peaks which indicate that phonons may be emitted at intermediate angles for precise values of $E_{q}$ (e.g., TA-PZ phonons at $\hbar \omega_{0}=3.37 \mathrm{meV}$ ). Actually, one can notice a clear correspondence between the position of these peaks and the minima of the emission rates in Fig. 1(b). The origin of these scattering minima is known to be the formation of antiphase relations

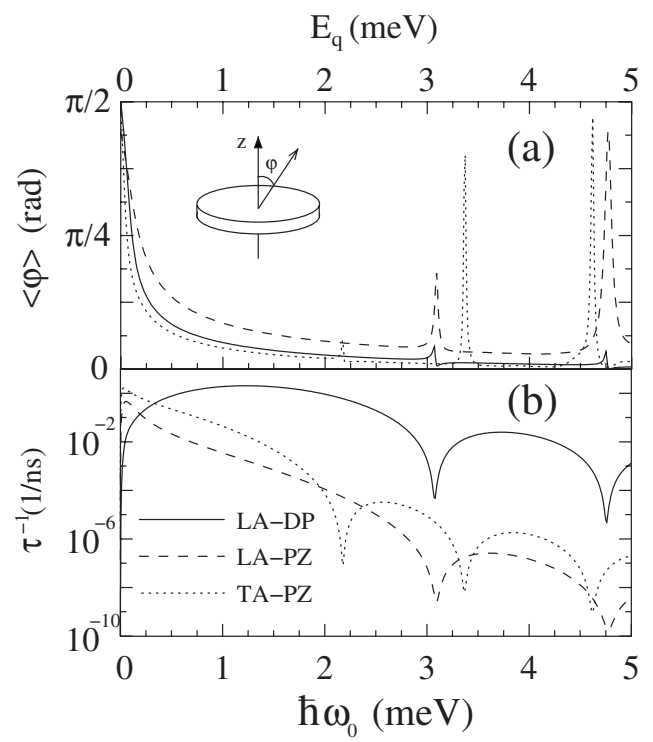

FIG. 1. Mean phonon emission angle (a) and scattering rate (b) in a QD with height $L_{z}=10 \mathrm{~nm}$ as a function of the lateral confinement frequency $\hbar \omega_{0}$. Different lines are used for LA and TA phonons coming from DP and PZ interaction. The electron transition is $(n, m, g)=(0,1,0) \rightarrow(0,0,0)$. Top axis: energy of the emitted phonon. The inset in (a) defines the emission angle $\varphi$. 

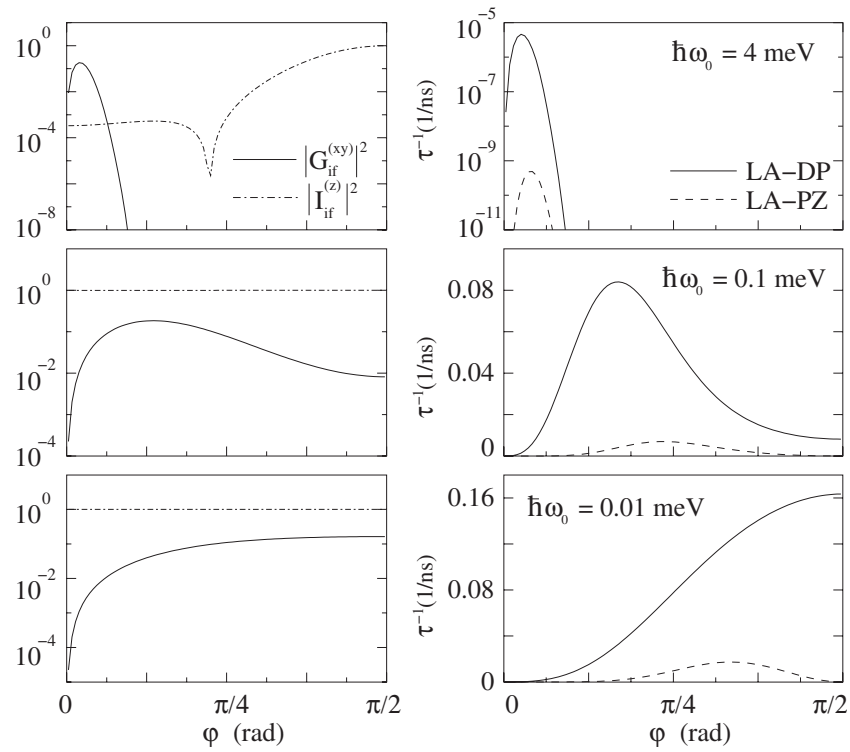

FIG. 2. Left column: in-plane and vertical components of the squared structure factor (dimensionless units) vs phonon emission angle. Right column: emission rate of LA phonons due to DP and PZ interaction vs emission angle. The electron transition considered is $(n, m, g)=(0,1,0) \rightarrow(0,0,0)$. The QDs have height $L_{z}=10 \mathrm{~nm}$ and the lateral confinement frequency indicated in the right panel. Note that $E_{q}=\hbar \omega_{0}$.

between the phonon wave and the electron wave function along the growth direction. ${ }^{6-8,12}$ At these specific points, phonon emission along the growth direction is greatly suppressed and, since it was the preferential emission direction, $\langle\varphi\rangle$ is abruptly tilted. This tilting is much more sizeable for PZ phonons than for DP ones, because only the former depend explicitly on the individual phonon momentum projections.

In order to understand the physical mechanisms which determine the phonon emission angle, in Fig. 2 we investigate the $\varphi$ dependence of the in-plane and vertical structure factor components (left column), as well as that of the emission rate of LA-DP and LA-PZ phonons (right column). The structure factors provide an insight on the electron-phonon coupling efficiency on the QD length scale, while the total emission rate adds the matrix elements $M_{\nu}(\mathbf{q})$ contribution, so that the effects of the host semiconductor crystal are also included.

Three different regimes of phonon energy are shown in the figure: high (top panels), intermediate (middle panels), and low (bottom panels). For high phonon energies, since the phonon momentum $q_{0}$ is fairly large, the structure factors components depend strongly on $\varphi$, and it is mainly these that determine the preferential phonon emission angle. This is seen in the top panels of Fig. 2, where both $\left|G_{i f}^{(x y)}\left(q_{0}, \varphi\right)\right|^{2}$ and $\left|I_{i f}^{(z)}\left(q_{0}, \varphi\right)\right|^{2}$ change by several orders of magnitude with $\varphi$. Indeed, the preferential emission angle is found at small but finite values of $\varphi$ because $\left|G_{i f}^{(x y)}\left(q_{0}, \varphi\right)\right|^{2}$ rapidly vanishes for $\varphi>\pi / 8$ and at $\varphi=0$. This can be understood as follows. For few-meV phonon energy, the wavelength of the phonon is of the order of the QD height, but much shorter than the QD lateral size. ${ }^{12}$ Therefore unless the in-plane projection of

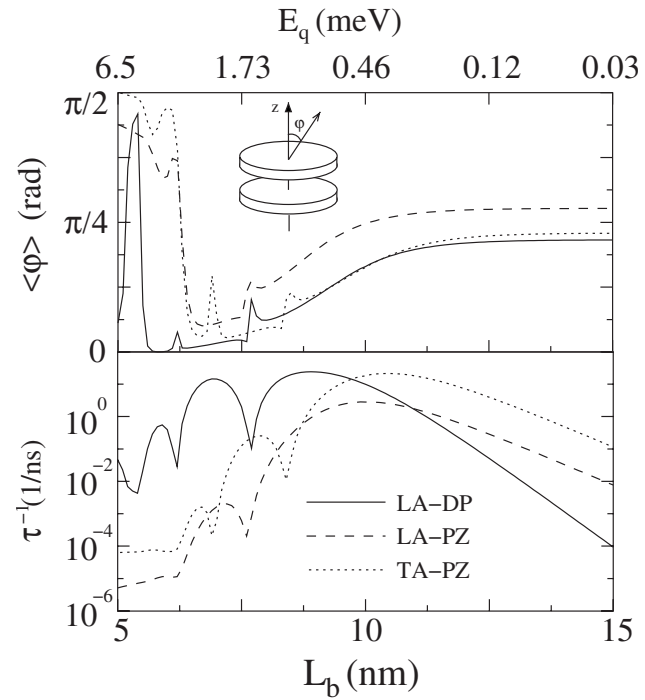

FIG. 3. Mean phonon emission angle (a) and scattering rate (b) vs interdot barrier thickness in a CQD. The CQDs have height $L_{z}$ $=10 \mathrm{~nm}$ and lateral confinement $\hbar \omega_{0}=5 \mathrm{meV}$. Different lines are used for LA and TA phonons due to DP and PZ interaction. The electron transition is $(n, m, g)=(0,0,1) \rightarrow(0,0,0)$. The top axis shows the phonon energy, which corresponds to the bondingantibonding energy splitting. The inset in (a) defines the emission angle $\varphi$.

the phonon momentum is small, in the lateral direction the phonon oscillation period is much shorter than the length scale of the electron wave function. This makes $\left|G_{i f}^{(x y)}\left(q_{0}, \varphi\right)\right|^{2}$ vanish, because the initial and final electron states do not possess the proper Fourier component to trigger the phonon oscillation, and they are orthogonal to each other. On the other hand, when the phonon momentum in-plane projection is negligible, the phonon wave is almost constant in the length scale of the electron state, and thus the orthogonality of the initial and final states again yield reduced values of $\left|G_{i f}^{(x y)}\left(q_{0}, \varphi\right)\right|^{2}$. However, for the typical lateral sizes of weakly confined QDs this holds strictly only in the near vicinity of $\varphi=0$. Finally, we note that $\left|I_{i f}^{(z)}\left(q_{0}, \varphi\right)\right|^{2}$ does not show any vanishing limit in intradot transitions, because the initial and final states of the quantum well, $\xi_{0}$, are not orthogonal. The dip in the top left panel corresponds to an antiphase relation of the phonon wave $z$ projection and the electron wave function in the quantum well. ${ }^{12}$

A different situation is found in the regime of low phonon energies (bottom panels). In this case, the phonon wavelength is no longer short as compared to the lateral dimension of the dot, so that the in-plane structure factor does not reach the orthogonality limit for large values of $\varphi$. Moreover, due to the small value of $q_{0}$, the structure factors only weakly depend on the emission angle, and the preferential direction of emission is mostly given by the angular dependences arising from the scattering matrix elements $M_{\nu}(\mathbf{q})$ and the infinitesimal element of volume in Eqs. (9). Thus this is the regime where the propagation direction of phonons coming from DP and PZ interactions differ the most. For DP interaction, the preferential emission can be in the QD plane (see bottom right panel), while for $\mathrm{PZ}$ interaction this is forbidden 

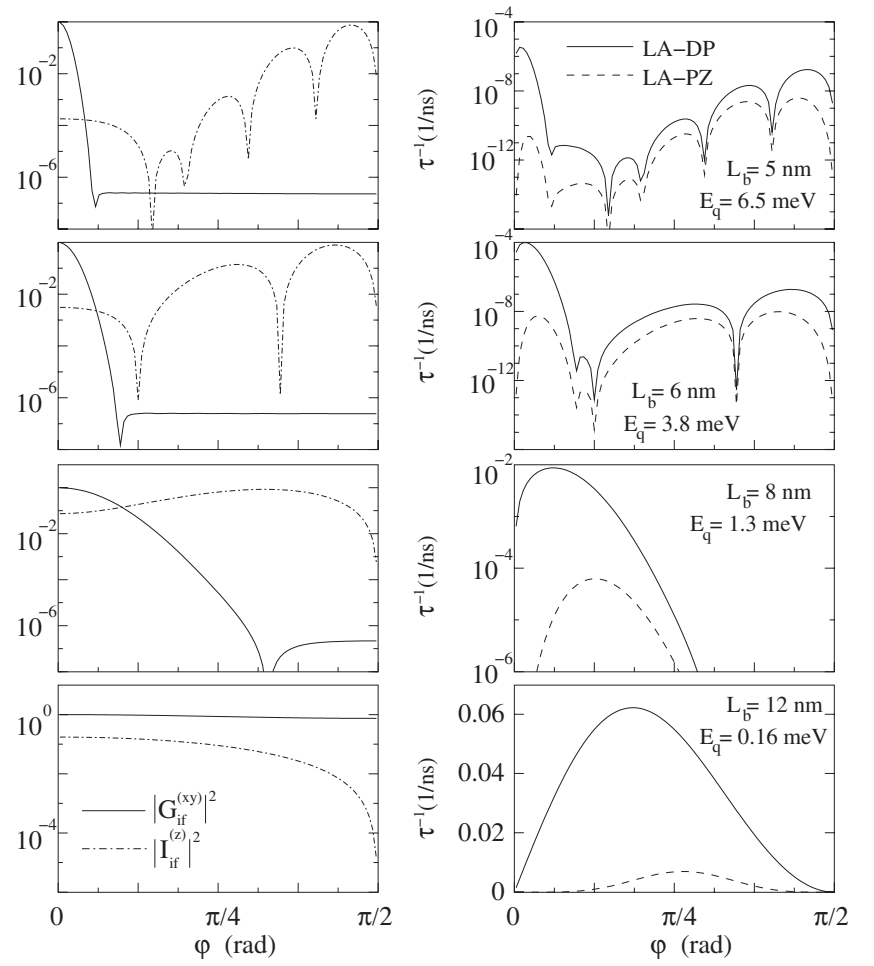

FIG. 4. Left column: in-plane and vertical components of the squared structure factor (dimensionless units) vs phonon emission angle. Right column: emission rate of LA phonons coming from DP and PZ interaction vs emission angle. The electron transition considered is $(n, m, g)=(0,0,1) \rightarrow(0,0,0)$. The CQDs have height $L_{z}$ $=10 \mathrm{~nm}$ and lateral confinement $\hbar \omega_{0}=5 \mathrm{meV}$. The interdot barrier thickness and the corresponding phonon energy are indicated in the right panel.

because the matrix element is proportional to $q_{z}$ (which is zero for in-plane emission).

\section{PHONON EMISSION IN COUPLED QUANTUM DOTS}

Next, we investigate the emission of phonons by electronscattering between molecular states of vertically CQDs. Figure 3 illustrates the phonon emission rate and mean emission angle due to the different electron-phonon interaction mechanisms, as a function of the interdot barrier thickness $L_{b}$. Note that the energy of the emitted phonon (top axis), which corresponds to the tunneling energy, decreases as the barrier thickness (bottom axis) increases.

Several differences in the phonon emission angle are found as compared to the previous section. In the high phonon-energy regime (small barrier thickness) phonon emission may take place close to the in-plane direction $(\varphi$ $\sim \pi / 2$ ), while for intermediate energies it switches to the growth direction $(\varphi \sim 0)$ abruptly and finally, for low energies (large barrier thickness), phonon propagation takes place at intermediate angles $(\varphi \sim \pi / 4)$. Peaks of the mean emission angle at the position of scattering rate minima are found in analogy with the intradot case.

In order to understand the different behavior of the phonons coming from interdot transitions, in Fig. 4 we ana- lyze the $\varphi$ dependence of the structure factor components (left column) and the emission rate of LA phonons due to DP and PZ interactions (right column) for different regimes: high (top panels), intermediate (middle panels), and low (bottom panels) phonon energy. The dependence of the structure factors on the emission angle is clearly different from that of Fig. 2. The underlying reason is the different symmetries of the electron states involved in the interdot transition as compared to the intradot ones: here the initial and final FD states are no longer orthogonal. As a result, $\left|G_{i f}^{(x y)}\left(q_{0}, \varphi\right)\right|^{2}$ does not vanish at $\varphi=0$. In contrast, the initial and final quantum well solutions have now become orthogonal, so that $\left|I_{i f}^{(z)}\left(q_{0}, \varphi\right)\right|^{2}$ may eventually vanish in the limits of vertical or in-plane emission.

In the high-energy regime, for $\varphi \sim 0$, the in-plane projection of the phonon momentum is negligible and then $\left|G_{i f}^{(x y)}\left(q_{0}, \varphi\right)\right|^{2}$ yields simply the overlap integral of the lateral electronic states. As the emission angle increases, the phonon oscillation period in the plane becomes rapidly short as compared to the lateral length scale of the QD. Then, the FD states lack the proper Fourier components to trigger phonon oscillation and $\left|G_{i f}^{(x y)}\left(q_{0}, \varphi\right)\right|^{2}$ is approximately the factorized product between the FD states overlap integral and the average value of the phonon wave in-plane projection, which is quite small. As for the analysis of $\left|I_{i f}^{(z)}\left(q_{0}, \varphi\right)\right|^{2}$, we note that the regular minima vs $\varphi$ are signatures of the antiphase relation between the phonon and electron waves on the growth direction. ${ }^{12}$ Besides, the suppression due to orthogonality is attained only in the $\varphi=\pi / 2$ limit, when the vertical projection of the phonon wave vector is constant in the length scale of the quantum well. Conversely, for $\varphi \sim 0$, no suppression is observed. This is because the phonon wavelength is of the order of the QD height, so the phonon oscillation period is never short as compared to $\xi_{g}$. This constitutes an important difference with respect to the corresponding intradot transition case, where the short oscillation period of the phonon wave as compared to the electron lateral orbitals prevented emission close to the in-plane direction. Here, the preferential emission angle is determined by the balance between $\left|G_{i f}^{(x y)}\left(q_{0}, \varphi\right)\right|^{2}$ (which still favors emission along the growth direction) and $\left|I_{i f}^{(z)}\left(q_{0}, \varphi\right)\right|^{2}$ (which tends to favor emission near the in-plane direction).

When the phonon energy decreases (middle panels), the structure factor components become smoother and flatterer. $\left|I_{i f}^{(z)}\left(q_{0}, \varphi\right)\right|^{2}$ displays ever less oscillations because the phonon wavelength becomes large as compared to the the quantum well width, and it flattens faster than $\left|G_{i f}^{(x y)}\left(q_{0}, \varphi\right)\right|^{2}$ because of the strong vertical confinement. Hence in this regime the angle of emission is mostly determined by the electron-phonon coupling in the lateral direction. Finally, for low phonon energies (bottom panels), $\left|G_{i f}^{(x y)}\left(q_{0}, \varphi\right)\right|^{2}$ is simply the overlap integral between the FD states, and $\left|I_{i f}^{(z)}\left(q_{0}, \varphi\right)\right|^{2}$ is constant except in the vicinity of the $\varphi$ $\sim \pi / 2$ vanishing limit. This prevents emission close to the in-plane direction, as would be the case for intradot transitions. Moreover, the main emission angle is to a large extent determined by the scattering matrix elements, $M_{\nu}(\mathbf{q})$. 


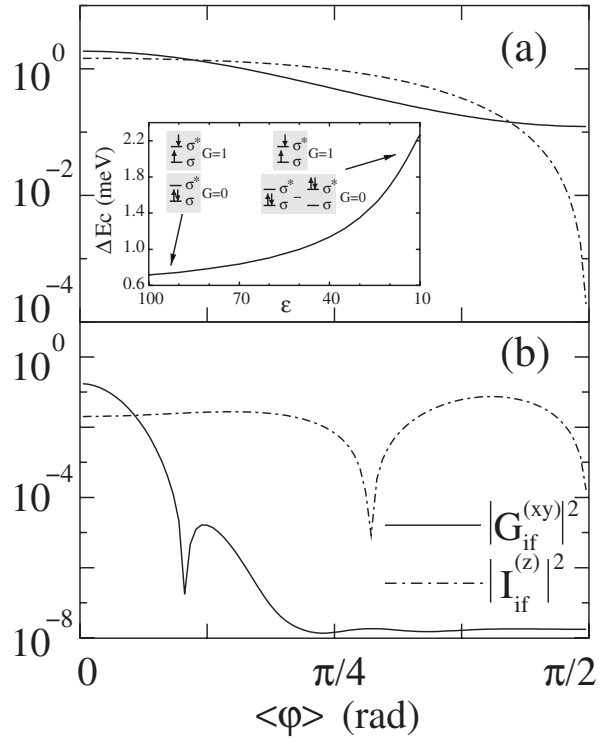

FIG. 5. In-plane and vertical components of the squared structure factor (dimensionless units) vs. phonon emission angle for the $(N, M, G)=(0,0,1) \rightarrow(0,0,0)$ two-electron transition in a CQD without (a) and with (b) Coulomb interaction. The CQDs have height $L_{z}=10 \mathrm{~nm}$, lateral confinement $\hbar \omega_{0}=5 \mathrm{meV}$, and interdot barrier $L_{b}=10 \mathrm{~nm}$. The inset in (a) depicts the energy difference between the lowest $G=1$ and $G=0$ states as the Coulomb interaction increases, as well as the most important configurations in each limit.

\section{SUPPRESSION OF PIEZOELECTRIC SCATTERING IN TWO-ELECTRON CQDS}

We have recently predicted the suppression of the PZ contribution to charge relaxation in two-electron interdot transitions. ${ }^{10}$ This result may be of technological interest, for example in the implementation of so-called isospin qubits, ${ }^{18}$ because it suggests that two-electron states with long lifetimes may be achieved in vertically CQDs using thinner barriers than for single-electron states. It was shown that the origin of this suppression was related to the phonon emission angle, which in the presence of Coulomb interaction lies very close to the growth direction, and thus renders the PZ scattering matrix element small [recall that $M_{P Z}(\mathbf{q})$ depends explicitly on $q_{x}$ and $q_{y}$, Eqs. (5) and (6)]. Here we analyze in more detail this phenomenon, by relating it with the behavior of interdot transitions discussed above. Thus in Fig. 5 we depict $\left|G_{i f}^{(x y)}\left(q_{0}, \varphi\right)\right|^{2}$ and $\left|I_{i f}^{(z)}\left(q_{0}, \varphi\right)\right|^{2}$ against $\varphi$ for the fundamental interdot transition of two electrons in a CQD with $L_{b}=10 \mathrm{~nm}$. The top (bottom) panel illustrates the case without (with) Coulomb interaction. The interacting electron states have been calculated using the configuration interaction approach described in Ref. 10, and they can be classified as the $N$ th state with total angular momentum $M$ and total parity $G$.

Clearly, the structure factor components of the noninteracting case correspond to the low-energy limit of Fig. 4. On the contrary, the interacting picture resembles the highphonon-energy limit, with emission close to the vertical di- rection of the CQD. An inspection of the phonon energy vs the effective dielectric constant (inset of Fig. 5) confirms this observation: for small dielectric constant (strongly interacting limit) the phonon energy is enhanced as compared to the large dielectric constant regime (weakly interacting limit). This is because in the presence of Coulomb interaction, the dominant configurations of the two-electron $G=0$ state is given by a linear combination of two electrons in the lowest bonding orbital and two in the lowest antibonding one $\left(\xi_{0}^{2}\right.$ $-\xi_{1}^{2}$ ) (see diagrammatic scheme in the inset). Electronic correlations reduce the Coulomb energy of this state as compared to the $G=1$ one, where no such mixing is possible. Therefore the stronger the Coulomb interaction the larger the difference in electron-electron repulsion energy between the $G=0$ and $G=1$ states. Note that this behavior is characteristic of two-electron systems. For instance, for three electrons the main configuration of the lowest $G=1$ state would be $\left(\xi_{0}^{2} \xi_{1}\right)$, and that of the $G=1$ state $\left(\xi_{0} \xi_{1}^{2}\right)$. Hence the unpaired electrons prevent the formation of strongly correlated states while preserving the parity symmetry. We then conclude that, for realistic values of the semiconductor dielectric constant, PZ interaction in two-electron interdot transitions is quenched by the large correlation-induced phonon energies, which in turn force phonon emission in the vicinity of the growth direction.

\section{CONCLUSIONS}

We have investigated the direction of propagation of acoustic phonons due to electron energy relaxation in single and vertically coupled parabolic QDs. For high-energy intradot transitions, phonon emission takes place mainly along the vertical direction, and it switches to in-plane angles for low-energy transitions. Instead, for transitions between bonding and antibonding states of vertically CQDs, in-plane emission may prevail in the high-energy limit, while emission mostly occurs at intermediate angles in the low-energy limit. Differences in the direction of propagation of phonons arising from DP and PZ interactions have been reported, the differences being particularly pronounced in the low-energy limit. We have also explained recent theoretical results, which predicted quenched PZ scattering in two-electron vertically CQD systems, ${ }^{10}$ in terms of the anisotropic phonon emission and correlation effects.

The energy dependence of the phonon emission direction we have described here is a general result of weakly confined, disk-shaped GaAs/AlGaAs QDs. We have exemplified it in cases where the phonon energy was varied by means of structural parameters (QD confinement potential or interdot barrier), but it also holds if it is varied with external fields, ${ }^{7}$ which would certainly be a flexible way to control the phonon emission angle.

Finally, we mention that the main results reported here are not exclusive for the symmetric confinement potentials we have assumed. In realistic samples, where lateral and vertical symmetries may be broken, the factors determining the phonon emission direction are essentially the same we have described (orthogonality of the initial and final electron states, interplay between the phonon and QD length scales). Mod- 
erate quantitative discrepancies may of course be expected, due to changes in the energy structure and the QD geometry. Furthermore, in-plane phonon emission would no longer be isotropic, but this effect should be small because in usual elliptical QDs the confinement in $x$ and $y$ is of the same order.

\section{ACKNOWLEDGMENTS}

The authors thank Massimo Rontani for fruitful discussions. Support from the Italian Ministry for University and Research under FIRB RBIN04EY74, and EU under Marie Curie IEF project MEIF-CT-2006-023797 is acknowledged.
*Electronic address: climente@unimore.it; URL: http:// www.nanoscience.unimo.it/

${ }^{1}$ T. Fujisawa, D. G. Austing, Y. Tokura, Y. Hirayama, and S. Tarucha, Nature (London) 419, 278 (2002); J. Phys.: Condens. Matter 15, R1395 (2003).

${ }^{2}$ S. Tarucha, T. Fujisawa, K. Ono, D. G. Austing, T. H. Oosterkamp, W. G. van der Wiel, and L. P. Kouwenhoven, Microelectron. Eng. 47, 101 (1999).

${ }^{3}$ G. Ortner, R. Oulton, H. Kurtze, M. Schwab, D. R. Yakovlev, M. Bayer, S. Fafard, Z. Wasilewski, and P. Hawrylak, Phys. Rev. B 72, 165353 (2005).

${ }^{4}$ T. Nakaoka, E. C. Clark, H. J. Krenner, M. Sabathil, M. Bichler, Y. Arakawa, G. Abstreiter, and J. J. Finley, Phys. Rev. B 74, 121305(R) (2006).

${ }^{5}$ U. Bockelmann, Phys. Rev. B 50, 17271 (1994).

${ }^{6}$ P. Zanardi and F. Rossi, Phys. Rev. Lett. 81, 4752 (1998).

${ }^{7}$ A. Bertoni, M. Rontani, G. Goldoni, F. Troiani, and E. Molinari, Appl. Phys. Lett. 85, 4729 (2004).

${ }^{8}$ J. I. Climente, A. Bertoni, G. Goldoni, and E. Molinari, Phys. Rev. B 74, 035313 (2006), and references therein.

${ }^{9}$ M. Dur, A. D. Gunther, D. Vasileska, and S. M. Goodnick, Nano- technology 10, 142 (1999).

${ }^{10}$ J. I. Climente, A. Bertoni, M. Rontani, G. Goldoni, and E. Molinari, Phys. Rev. B 74, 125303 (2006).

${ }^{11}$ O. L. Lazarenkova and A. A. Balandin, Phys. Rev. B 66, 245319 (2002).

${ }^{12}$ A. Bertoni, M. Rontani, G. Goldoni, F. Troiani, and E. Molinari, Physica E (Amsterdam) 26, 427 (2005).

${ }^{13}$ P. Stano and J. Fabian, Phys. Rev. B 74, 045320 (2006).

${ }^{14}$ L. Jacak, P. Hawrylak, and A. Wójs, Quantum Dots (SpringerVerlag, Berlin, 1998).

${ }^{15}$ Semiconductors. Physics of Group IV Elements and III-V Compounds, edited by O. Madelung, Landolt-Börnstein, Vol. 17 (Springer-Verlag, 1982).

${ }^{16}$ Physics of Hot Electron Transport in Semiconductors, edited by C. S. Ting (World Scientific, Singapore, 1992).

${ }^{17}$ The general behavior observed in Fig. 1 agrees with that predicted in Ref. 5. In particular, we confirm that the agreement is valid at small phonon energies, where PZ interaction (neglected in Ref. 5) is actually the dominant scattering mechanism.

${ }^{18}$ D. Jacob, B. Wunsch, and D. Pfannkuche, Phys. Rev. B 70, 081314(R) (2004). 